Canad. Math. Bull. Vol. 49 (3), 2006 pp. 472-480

\title{
Cyclic Cubic Fields of Given Conductor and Given Index
}

\author{
Alan K. Silvester, Blair K. Spearman, and Kenneth S. Williams
}

Abstract. The number of cyclic cubic fields with a given conductor and a given index is determined.

\section{Introduction}

Let $K$ be a cyclic cubic extension of $(\mathbb{O})$ so that $[K:(\mathbb{Q}]=3$ and $\mathrm{Gal}(K / \mathbb{O}) \simeq \mathbb{Z} / 3 \mathbb{Z}$. By the Kronecker-Weber theorem [10, p. 289] there exists a positive integer $m$ such that the cyclotomic field $\mathbb{O})\left(e^{2 \pi i / m}\right) \supseteq K$. The smallest such $m$ is called the conductor of $K$ and is denoted by $f(K)$. The discriminant of $K$ is given by $d(K)=f(K)^{2}[8$, p. 831]. The conductor $f(K)$ of a cyclic cubic field is of the form

$$
f=p_{1} p_{2} \cdots p_{r}
$$

where $r \in \mathbb{N}$ and $p_{1}, \ldots, p_{r}$, are distinct integers from the set

$$
P=\{9\} \cup\{p(\text { prime }) \equiv 1(\bmod 3)\}=\{7,9,13,19,31,37, \ldots\},
$$

see $[8$, p. 831]. Moreover each positive integer $f$ of the form (1.1) is the conductor of some cyclic cubic field; indeed it is the conductor of $2^{r-1}$ cyclic cubic fields [8, p. 831]. For any cubic field $K$ it is known that its field index $i(K)=1$ or 2 [5, p. 234]. For $f$ of the form (1.1) and $i \in\{1,2\}$, we define

$$
N(f, i)=\text { number of cyclic cubic fields } K \text { with } f(K)=f \text { and } i(K)=i,
$$

so that

$$
N(f, 1)+N(f, 2)=2^{r-1} .
$$

In this paper we determine $N(f, 1)$ and $N(f, 2)$.

It is well known that each prime $p \equiv 1(\bmod 3)$ has a unique representation in the form

$$
4 p=a^{2}+27 b^{2}, \quad a, b \in \mathbb{N},
$$

Received by the editors July 2, 2004; revised July 16, 2006.

The second and third authors were supported by grants from the Natural Sciences and Engineering Research Council of Canada.

AMS subject classification: Primary: 11R16; secondary: 11R29.

Keywords: Discriminant, conductor, index, cyclic cubic field.

(C) Canadian Mathematical Society 2006. 
see [1, Theorem 3.1.3, p. 105; Lemma 3.0.1, p. 101]. Clearly for such a representation we have $a \equiv b(\bmod 2)$ and

$$
\operatorname{gcd}(a, b)=1 \text { or } 2 \text {. }
$$

It is a classical result of Gauss that 2 is a cubic residue $(\bmod p)$ if and only if $\operatorname{gcd}(a, b)=2$, see [1, Theorem 7.1.1, p. 213]. We set

$$
P_{1}=\{9\} \cup\left\{p(\text { prime }) \equiv 1(\bmod 3), 4 p=a^{2}+27 b^{2}, \operatorname{gcd}(a, b)=1\right\}
$$

and

$$
P_{2}=\left\{p(\text { prime }) \equiv 1(\bmod 3), 4 p=a^{2}+27 b^{2}, \operatorname{gcd}(a, b)=2\right\}
$$

so that

$$
P_{1} \cup P_{2}=P, \quad P_{1} \cap P_{2}=\phi .
$$

Clearly

$$
P_{1}=\{7,9,13,19,37, \ldots\}, \quad P_{2}=\{31,43,109,127, \ldots\} .
$$

If $p$ is a prime in $P_{1}$, then $a \equiv b \equiv 1(\bmod 2)$. Replacing $b$ by $-b$, if necessary, we may suppose that $a \equiv b(\bmod 4)$. Set $x=(a-b) / 4 \in \mathbb{Z}, y=b \in \mathbb{Z}$. Then $4 x^{2}+2 x y+7 y^{2}=p$. Conversely if $p=4 x^{2}+2 x y+7 y^{2}$ for some $x, y \in \mathbb{Z}$ then $y$ is odd, $\operatorname{gcd}(x, y)=1$ and $4 p=a^{2}+27 b^{2}$ with $a=|4 x+y|, b=|y|$ and $\operatorname{gcd}(a, b)=$ $\operatorname{gcd}(4 x+y, y)=\operatorname{gcd}(4 x, y)=\operatorname{gcd}(x, y)=1$. Thus the primes in $P_{1}$ are precisely those which can be expressed in the form $4 x^{2}+2 x y+7 y^{2}$ for some $x, y \in \mathbb{Z}$. The primes in $P_{2}$ are precisely those which can be expressed in the form $x^{2}+27 y^{2}$ for some $x, y \in \mathbb{Z}$.

Now suppose that $f$ is of the form (1.1) with

$$
p_{1}, p_{2}, \ldots, p_{u} \in P_{1} \quad \text { and } \quad p_{u+1}, p_{u+2}, \ldots, p_{r} \in P_{2}
$$

where $u \in\{0,1, \ldots, r\}$. In Section 5 we prove the following result.

Theorem With the above notation, we have

$$
N(f, 1)=\frac{1}{3}\left(2^{r}-(-1)^{u} 2^{r-u}\right), \quad N(f, 2)=\frac{1}{3}\left(2^{r-1}+(-1)^{u} 2^{r-u}\right) .
$$

In Sections 2, 3, 4 we give some results on representations of integers by binary quadratic forms which will be needed in the proof of this theorem. 


\section{The Form Class Group $H(d)$}

Let $H(d)$ denote the set of classes of primitive, positive-definite, integral binary quadratic forms $(a, b, c)=a x^{2}+b x y+c y^{2}$ of discriminant $d=b^{2}-4 a c \equiv 0$ or $1(\bmod 4)$ under the action of the modular group. As $a x^{2}+b x y+c y^{2}$ is positive-definite, we have $a>0$ and $d<0$. The class of the form $(a, b, c)$ is denoted by $[a, b, c]$. Multiplication of classes of $H(d)$ is due to Gauss and is described, for example, in [2]. With respect to multiplication, $H(d)$ is a finite abelian group called the form class group of discriminant $d$. The order of $H(d)$ is called the form class number of discriminant $d$ and is denoted by $h(d)$. The identity $I$ of the group $H(d)$ is the principal class

$$
I= \begin{cases}{[1,0,-d / 4]} & \text { if } d \equiv 0(\bmod 4), \\ {[1,1,(1-d) / 4]} & \text { if } d \equiv 1(\bmod 4)\end{cases}
$$

The inverse of the class $K=[a, b, c] \in H(d)$ is the class $K^{-1}=[a,-b, c] \in H(d)$. Each class of $H(d)$ contains one and only one form $(a, b, c)$ with

$$
-a<b \leq a \leq c, \quad b \geq 0 \text { if } a=c, \quad b^{2}-4 a c=d, \operatorname{gcd}(a, b, c)=1,
$$

see [4, pp. 68-71]. Let $n \in \mathbb{N}$. If $x$ and $y$ are integers such that $n=a x^{2}+b x y+c y^{2}$, then $(x, y)$ is called a representation of the positive integer $n$ by the form $(a, b, c)$. As $(a, b, c)$ is a positive-definite form, the number $R_{(a, b, c)}(n)$ of representations of $n$ by the form $(a, b, c)$ is finite. If in addition the representation $(x, y) \operatorname{satisfies} \operatorname{gcd}(x, y)=$ 1 , then the representation is called primitive. The number of primitive representations of $n$ by the form $(a, b, c)$ is denoted by $P_{(a, b, c)}(n)$. Clearly,

$$
R_{(a, b, c)}(n)=\sum_{e^{2} \mid n} P_{(a, b, c)}\left(n / e^{2}\right)
$$

If $(A, B, C)$ is a form equivalent to $(a, b, c)$ it is well known that $R_{(A, B, C)}(n)=R_{(a, b, c)}(n)$ and $P_{(A, B, C)}(n)=P_{(a, b, c)}(n)$. Hence we can define the number of representations of $n \in \mathbb{N}$ by the class $K \in H(d)$ by

$$
R_{K}(n)=R_{(a, b, c)}(n) \text { for any }(a, b, c) \in K
$$

and the number of primitive representations of $n \in \mathbb{N}$ by the class $K \in H(d)$ by

$$
P_{K}(n)=P_{(a, b, c)}(n) \text { for any }(a, b, c) \in K \text {. }
$$

From (2.2)-(2.4) we deduce that for $n \in \mathbb{N}$ and $K \in H(d)$

$$
R_{K}(n)=\sum_{e^{2} \mid n} P_{K}\left(n / e^{2}\right) .
$$

In particular, if $n \in \mathbb{N}$ is squarefree, we have

$$
R_{K}(n)=P_{K}(n)
$$


As each representation $(x, y)$ of $n$ by $(a, b, c)$ gives a representation $(x,-y)$ of $n$ by $(a,-b, c)$ and conversely, we have for $n \in \mathbb{N}$ and $K \in H(d)$

$$
R_{K}(n)=R_{K^{-1}}(n), \quad P_{K}(n)=P_{K^{-1}}(n) .
$$

For $n_{1}, n_{2} \in \mathbb{N}$ with $n_{1}$ squarefree, $n_{2}$ squarefree and $\operatorname{gcd}\left(n_{1}, n_{2}\right)=1$, it is known that

$$
R_{K}\left(n_{1} n_{2}\right)=\frac{1}{w(d)} \sum_{K_{1} K_{2}=K} R_{K_{1}}\left(n_{1}\right) R_{K_{2}}\left(n_{2}\right),
$$

where $K_{1}, K_{2}$ run through all the classes of $H(d)$ whose product is $K$, and

$$
w(d)=6,4 \text { or } 2 \text { according as } d=-3, d=-4 \text { or } d<-4,
$$

see [9, (29) and Lemma 5.5]. The largest positive integer $f$ such that $f^{2} \mid d$ with $\Delta=d / f^{2} \equiv 0$ or $1(\bmod 4)$ is called the conductor of $d$. By a theorem of Dirichlet, see [6], we have for $\operatorname{gcd}(n, f)=1$

$$
\sum_{K \in H(d)} R_{K}(n)=w(d) \sum_{e \mid n}\left(\frac{d}{e}\right)=w(d) \sum_{e \mid n}\left(\frac{\Delta}{e}\right)
$$

where $\left(\frac{d}{*}\right)$ is the Legendre-Jacobi-Kronecker symbol of discriminant $d$. If $p$ is a prime such that $\left(\frac{d}{p}\right)=1$, then there is at least one class $C \in H(d)$ which represents $p$. If $C=C^{-1}$, then $C$ is the only class of $H(d)$ representing $p$ and $R_{C}(p)=2 w(d)$. If $C \neq C^{-1}$, then $C$ and $C^{-1}$ are the only classes of $H(d)$ representing $p$ and $R_{C}(p)=$ $R_{C^{-1}}(p)=w(d)$. See $[9$, Lemma 5.3].

\section{Representations of Integers by $[1,0,3]$}

From (2.1) with $d=-12$ we find

$$
H(-12)=\{I\}, \quad h(-12)=1,
$$

where

$$
I=[1,0,3]
$$

Here $f=2$ and $\Delta=-3$.

Lemma 3.1 Let $p_{1}, \ldots, p_{t}(t \geq 0)$ be distinct primes $\equiv 1(\bmod 3)$. Then

$$
\begin{aligned}
& R_{I}\left(p_{1} \cdots p_{t}\right)=2^{t+1}, \quad P_{I}\left(p_{1} \cdots p_{t}\right)=2^{t+1} \\
& R_{I}\left(9 p_{1} \cdots p_{t}\right)=2^{t+1}, \quad P_{I}\left(9 p_{1} \cdots p_{t}\right)=0 .
\end{aligned}
$$


Proof If $n \in \mathbb{N}$ is such that $\operatorname{gcd}(n, 2)=1$, by (2.9) and (2.10) with $d=-12$, we have

$$
R_{I}(n)=2 \sum_{e \mid n}\left(\frac{-3}{e}\right)
$$

Taking $n=p_{1} \cdots p_{t}$, as $\left(\frac{-3}{p_{i}}\right)=1(i=1, \ldots, t)$, we obtain

$$
R_{I}\left(p_{1} \cdots p_{t}\right)=2 \sum_{e \mid p_{1} \cdots p_{t}} 1=2 \cdot 2^{t}=2^{t+1} .
$$

Then, appealing to (2.6), we obtain

$$
P_{I}\left(p_{1} \cdots p_{t}\right)=2^{t+1} .
$$

Taking $n=9 p_{1} \cdots p_{t}$ in $(3.1)$, since $\left(\frac{-3}{3}\right)=0$ we obtain

$$
R_{I}\left(9 p_{1} \cdots p_{t}\right)=2 \sum_{e \mid 9 p_{1} \cdots p_{t}}\left(\frac{-3}{e}\right)=2 \sum_{e \mid p_{1} \cdots p_{t}}\left(\frac{-3}{e}\right)=2^{t+1} .
$$

Finally, by (2.5), we have

$$
R_{I}\left(9 p_{1} \cdots p_{t}\right)=P_{I}\left(9 p_{1} \cdots p_{t}\right)+P_{I}\left(p_{1} \cdots p_{t}\right)
$$

so that

$$
P_{I}\left(9 p_{1} \cdots p_{t}\right)=2^{t+1}-2^{t+1}=0 .
$$

This completes the proof of the lemma.

\section{Representations of Integers by $[1,0,27]$ and $[4,2,7]$}

From (2.1) with $d=-108$ we find

$$
H(-108)=\left\{I, A, A^{2}\right\} \simeq \mathbb{Z} / 3 \mathbb{Z}, \quad h(-108)=3,
$$

where

$$
I=[1,0,27], \quad A=[4,2,7], \quad A^{2}=[4,-2,7], \quad A^{3}=I .
$$

Here $f=6$ and $\Delta=-3$.

Let $p$ be a prime with $p \equiv 1(\bmod 3)$. Then

$$
\left(\frac{d}{p}\right)=\left(\frac{-108}{p}\right)=\left(\frac{-2^{2} \cdot 3^{3}}{p}\right)=\left(\frac{-3}{p}\right)=1,
$$

so that $p$ is represented by some class in $H(-108)$. If $p$ is represented by $I$, then (as $\left.I=I^{-1}\right) I$ is the only class representing $p$, and

$$
R_{I}(p)=4, \quad R_{A}(p)=R_{A^{2}}(p)=0 .
$$


If $p$ is represented by $A$ or $A^{2}$, then (as $A \neq A^{-1}$ ) the only classes of $H(-108)$ representing $p$ are $A$ and $A^{2}$, and

$$
R_{I}(p)=0, \quad R_{A}(p)=R_{A^{2}}(p)=2 .
$$

Now let $m$ be a product of distinct primes $\equiv 1(\bmod 3)$. By $(2.9)$ and $(2.10)$ we have

$$
R_{I}(m)+R_{A}(m)+R_{A^{2}}(m)=2 \sum_{e \mid m}\left(\frac{-3}{e}\right)=2^{\tau(m)+1},
$$

where $\tau(m)$ denotes the number of primes dividing $m$. As $R_{A}(m)=R_{A^{-1}}(m)=$ $R_{A^{2}}(m)$ by (2.7), we deduce that

$$
R_{A}(m)=R_{A^{2}}(m)=2^{\tau(m)}-\frac{1}{2} R_{I}(m)
$$

By (2.8) we have for $p \nmid m$

$$
R_{I}(p m)=\frac{1}{2}\left(R_{I}(p) R_{I}(m)+R_{A}(p) R_{A^{2}}(m)+R_{A^{2}}(p) R_{A}(m)\right) .
$$

Appealing to (4.1)-(4.4), we obtain

$$
R_{I}(p m)= \begin{cases}2 R_{I}(m) & \text { if } R_{I}(p)>0 \\ 2^{\tau(m)+1}-R_{I}(m) & \text { if } R_{A}(p)>0\end{cases}
$$

We now use (4.5) to prove the following result.

Lemma 4.1 Let $p_{1}, \ldots, p_{l}$ be $l(\geq 0)$ distinct primes $\equiv 1(\bmod 3)$, which are represented by $I=[1,0,27]$, and let $q_{1}, \ldots, q_{m}$ be $m(\geq 0)$ distinct primes $\equiv 1(\bmod 3)$, which are represented by $A=[4,2,7]$. Then

$$
\begin{aligned}
R_{I}\left(p_{1} \cdots p_{l} q_{1} \cdots q_{m}\right) & =\frac{1}{3}\left(2^{l+m+1}+(-1)^{m} 2^{l+2}\right) \\
R_{A}\left(p_{1} \cdots p_{l} q_{1} \cdots q_{m}\right) & =R_{A^{2}}\left(p_{1} \cdots p_{l} q_{1} \cdots q_{m}\right)=\frac{1}{3}\left(2^{l+m+1}-(-1)^{m} 2^{l+1}\right) .
\end{aligned}
$$


Proof By (4.5) we obtain

$$
\begin{aligned}
R_{I}\left(p_{1} \cdots\right. & \left.p_{l} q_{1} \cdots q_{m}\right) \\
& =2 R_{I}\left(p_{1} \cdots p_{l-1} q_{1} \cdots q_{m}\right) \\
& =2^{2} R_{I}\left(p_{1} \cdots p_{l-2} q_{1} \cdots q_{m}\right) \\
& =\cdots \\
& =2^{l} R_{I}\left(q_{1} \cdots q_{m}\right) \\
& =2^{l}\left(2^{m}-R_{I}\left(q_{1} \cdots q_{m-1}\right)\right) \\
& =2^{l}\left(2^{m}-2^{m-1}+R_{I}\left(q_{1} \cdots q_{m-2}\right)\right) \\
& =\cdots \\
& =2^{l}\left(2^{m}-2^{m-1}+2^{m-2}-\cdots+(-1)^{m-2} 2^{2}+(-1)^{m-1} R_{I}\left(q_{1}\right)\right) \\
& =2^{l}\left(2^{m}-2^{m-1}+2^{m-2}-\cdots+(-1)^{m-2} 2^{2}\right) \\
& =\frac{1}{3}\left(2^{l+m+1}+(-1)^{m} 2^{l+2}\right)
\end{aligned}
$$

as required. Then, by (4.3), we obtain

$$
\begin{aligned}
R_{A}\left(p_{1} \cdots p_{l} q_{1} \cdots q_{m}\right) & =2^{l+m}-\frac{1}{2}\left(\frac{1}{3}\left(2^{l+m+1}+(-1)^{m} 2^{l+2}\right)\right) \\
& =\frac{1}{3}\left(2^{l+m+1}-(-1)^{m} 2^{l+1}\right)
\end{aligned}
$$

as asserted.

\section{Proof of Theorem}

There is a one-to-one correspondence between cyclic cubic fields $K$ and triples $(a, b, f) \in \mathbb{N}^{3}$ with

$$
\begin{aligned}
& a^{2}+27 b^{2}=4 f, \quad \operatorname{gcd}(a, b)=1 \text { or } 2, \quad f=p_{1} \cdots p_{r}, \\
& r \in \mathbb{N}, \quad p_{1}, \ldots, p_{r} \in P, \quad p_{i} \neq p_{j} \quad(1 \leq i<j \leq r),
\end{aligned}
$$

see [3, Section 6.4.6, pp. 336-343]. The cyclic cubic field corresponding to the triple $(a, b, f)$ is $K=(0)(\theta)$, where $\theta^{3}-3 f \theta+f a=0$. The conductor of $K$ is $f$. The index of $K$ is

$$
i(K)= \begin{cases}2 & \text { if } a \text { is even } \\ 1 & \text { if } a \text { is odd }\end{cases}
$$

see [7, Theorem 4, p. 585]. If $a$ is even, then $b$ is even and $\left(\frac{a}{2}\right)^{2}+27\left(\frac{b}{2}\right)^{2}=f$ with $\operatorname{gcd}\left(\frac{a}{2}, \frac{b}{2}\right)=1$. Thus

$$
N(f, 2)=\frac{1}{4} P_{[1,0,27]}(f) .
$$


First suppose that $9 \nmid f$. We may suppose that $p_{1}, \ldots, p_{u} \in P_{1}$ (so they are represented by $[4,2,7])$ and $p_{u+1}, \ldots, p_{r} \in P_{2}$ (so they are represented by $[1,0,27]$ ) with $u \in\{0,1,2, \ldots, r\}$. Then, by (2.6) and Lemma 4.1 (with $l=r-u$ and $m=u$ ), we have

$$
P_{[1,0,27]}(f)=R_{[1,0,27]}(f)=\frac{1}{3}\left(2^{r+1}+(-1)^{u} 2^{r-u+2}\right),
$$

so that

$$
N(f, 2)=\frac{1}{3}\left(2^{r-1}+(-1)^{u} 2^{r-u}\right), \quad 9 \nmid f .
$$

Now suppose that $9 \mid f$. We may suppose that $p_{1}=9, p_{2}, \ldots, p_{u} \in P_{1}$ (so they are represented by $[4,2,7]$ ) and $p_{u+1}, \ldots, p_{r} \in P_{2}$ (so they are represented by $[1,0,27]$ ). As $9 \mid f$ we have

$$
f=x^{2}+27 y^{2} \Longleftrightarrow f / 9=(x / 3)^{2}+3 y^{2}
$$

so that

$$
R_{[1,0,27]}(f)=R_{[1,0,3]}(f / 9) .
$$

As $f / 9$ is squarefree, we have

$$
R_{[1,0,27]}(f / 9)=P_{[1,0,27]}(f / 9) .
$$

From (2.5) we deduce

$$
R_{[1,0,27]}(f)=P_{[1,0,27]}(f)+P_{[1,0,27]}(f / 9) .
$$

Thus

$$
P_{[1,0,27]}(f)=R_{[1,0,3]}(f / 9)-R_{[1,0,27]}(f / 9) .
$$

Appealing to Lemma 3.1 (with $t=r-1$ ) and Lemma 4.1 (with $l=r-u$ and $m=u-1)$, we obtain

$$
P_{[1,0,27]}(f)=2^{r}-\frac{1}{3}\left(2^{r}+(-1)^{u-1} 2^{r-u+2}\right)=\frac{1}{3}\left(2^{r+1}+(-1)^{u} 2^{r-u+2}\right)
$$

so that

$$
N(f, 2)=\frac{1}{3}\left(2^{r-1}+(-1)^{u} 2^{r-u}\right), \quad 9 \mid f .
$$

Finally, from (1.4), we obtain in both cases

$$
N(f, 1)=2^{r-1}-N(f, 2)=\frac{1}{3}\left(2^{r}-(-1)^{u} 2^{r-u}\right) .
$$

This completes the proof of the theorem. 


\section{References}

[1] B. C. Berndt, R. J. Evans and K. S. Williams, Gauss and Jacobi Sums. Wiley, New York, 1998.

[2] D. A. Buell, Binary Quadratic Forms. Classical Theory and Modern Computations. Springer-Verlag, New York, 1989.

[3] H. Cohen, A Course in Computational Algebraic Number Theory. Graduate Texts in Mathematics 138, Springer-Verlag, Berlin, 1993.

[4] L. E. Dickson, Introduction to the Theory of Numbers. Dover, New York, 1957.

[5] H. T. Engstrom, On the common index divisors of an algebraic field. Trans. Amer. Math. Soc. 32 (1930), no. 2, 223-237.

[6] P. Kaplan and K. S. Williams, On a formula of Dirichlet. Far East J. Math. Sci. 5 (1997), no. 1, 153-157.

[7] P. Llorente and E. Nart, Effective determination of the decomposition of the rational primes in a cubic field. Proc. Amer. Math. Soc. 87 (1983), no. 4, 579-585.

[8] D. C. Mayer, Multiplicities of dihedral discriminants. Math. Comp. 58 (1992), 831-847.

[9] H. Muzaffar and K. S. Williams, Evaluation of Weber's functions at quadratic irrationalities. JP J. Algebra Number Theory Appl. 4(2004), no. 2, 209-259.

[10] W. Narkiewicz, Elementary and Analytic Theory of Algebraic Numbers. Second edition. Springer-Verlag, Berlin, 1990.

Department of Mathematics and Statistics Okanagan University College

Kelowna, BC

V1V 1 V7

e-mail:mascdman@canada.com

bspearman@ouc.bc.ca
School of Mathematics and Statistics Carleton University

Ottawa, ON

K1S $5 B 6$

e-mail:williams@math.carleton.ca 